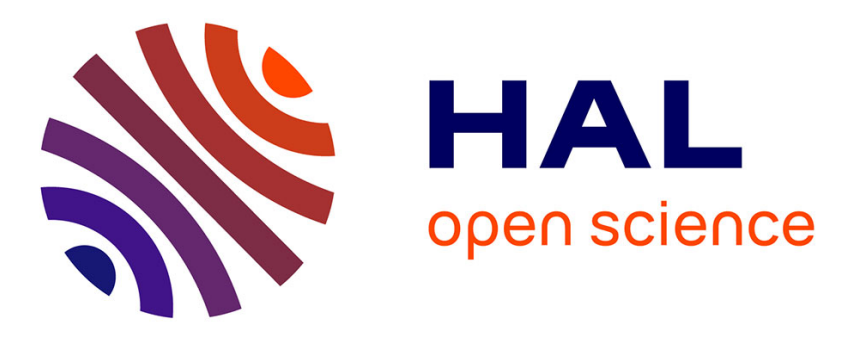

\title{
A Surface Enhanced Raman Spectroscopy study of aminothiophenol and aminothiophenol-C60 self-assembled monolayers: evolution of Raman modes with experimental parameters
}

Gregory Delafosse, Alexandre Merlen, Sylvain Clair, Lionel Patrone

\section{To cite this version:}

Gregory Delafosse, Alexandre Merlen, Sylvain Clair, Lionel Patrone. A Surface Enhanced Raman Spectroscopy study of aminothiophenol and aminothiophenol-C60 self-assembled monolayers: evolution of Raman modes with experimental parameters. Journal of Chemical Physics, 2012, 136, pp.194704. 10.1063/1.4717720 . hal-01007241

\section{HAL Id: hal-01007241 \\ https://hal.science/hal-01007241}

Submitted on 17 Jun 2014

HAL is a multi-disciplinary open access archive for the deposit and dissemination of scientific research documents, whether they are published or not. The documents may come from teaching and research institutions in France or abroad, or from public or private research centers.
L'archive ouverte pluridisciplinaire HAL, est destinée au dépôt et à la diffusion de documents scientifiques de niveau recherche, publiés ou non, émanant des établissements d'enseignement et de recherche français ou étrangers, des laboratoires publics ou privés. 
A Surface Enhanced Raman Spectroscopy study of aminothiophenol and aminothiophenol- $\mathrm{C}_{60}$ self-assembled monolayers: evolution of Raman modes with experimental parameters

\author{
G. Delafosse $e^{a, c, d}$, A. Merlen ${ }^{a, b^{*}}$, S. Clair ${ }^{a, c}$, L. Patrone $e^{a, c, d}$
}

${ }^{\text {a }}$ CNRS, IM2NP (UMR 7334)

${ }^{\mathrm{b}}$ Université du Sud Toulon-Var, IM2NP, Bâtiment R, BP 132, F-83957 La Garde Cedex, France

${ }^{c}$ Aix-Marseille Université, IM2NP, Campus de Saint-Jérôme - Case 142, F-13397 Marseille Cedex 20, France

${ }^{\text {d } I n s t i t u t ~ S u p e ́ r i e u r ~ d e ~ l ' E l e c t r o n i q u e ~ e t ~ d u ~ N u m e ́ r i q u e, ~ I M 2 N P, ~ M a i s o n ~ d e s ~ T e c h n o l o g i e s, ~ P l a c e ~}$ Georges Pompidou, F-83000 Toulon, France

* Electronic address: merlen@univ-tln.fr 
ABSTRACT: P-aminothiophenol (PATP) is a well known molecule for the preparation of self-assembled monolayers on gold via its thiol functional group. After adsorption, it has been demonstrated that this molecule is anchored to gold through its thiol group, and standing nearly upright at the surface with the amino functional group on top. This molecule has been extensively studied by Surface Enhanced Raman Spectroscopy but its exact SERS spectrum remains unclear. Here we demonstrate that it can be strongly affected by at least two experimental parameters: laser power and layer density. Those features are discussed in terms of a dimerization of the PATP molecules. The free amino group affords the adsorption of other molecules, like $\mathrm{C}_{60}$. In this case, a complex multilayer system is formed and the question of its precise characterisation remains a key point. In this article, we demonstrate that surface enhanced Raman spectroscopy combined with X-Ray photoelectron spectroscopy can bring very important information about the organisation of such a self-assembled multilayer on gold. In our study, the strong evolution of Raman modes after $\mathrm{C}_{60}$ adsorption suggests a change in the organisation of aminothiophenol molecules during $\mathrm{C}_{60}$ adsorption. These changes, also observed when the aminothiophenol layer is annealed in toluene, do not prevent the adsorption of $\mathrm{C}_{60}$ molecules.

Keywords: Self-assembled monolayers, surface enhanced Raman spectroscopy, aminothiophenol, fullerene $\mathrm{C}_{60}$. 


\section{INTRODUCTION}

The huge development of nanoelectronics leads to the elaboration of more and more complex architectures and to the subsequent difficult question of their physical and chemical characterisations. Among those architectures, self-assembled monolayers (SAM) on metallic nanostructures is an extremely promising approach, with potential applications in photovoltaics ${ }^{1}$, molecular electronics ${ }^{2}$, optics, and so on. Thiol functionalized molecules are known to form well-ordered, densely packed self-assembled monolayers on metals like gold or silver. The P-AminoThioPhenol (PATP) molecule appears as extremely promising due to its combination of electron-donating and electron-accepting groups, which is commonly designed as a "pushpull" molecule. In addition it is possible to elaborate "sandwich" PATP SAMs between gold substrate and another molecule using its respective two functional groups, i.e., thiol and amino groups. For instance, as for silver $^{3}$ or gold ${ }^{4}$ colloids, fullerene molecules ${ }^{5}$ can be in principle grafted on top of the terminal amine moieties of PATP self-assembled on gold as a stacked SAM. Indeed, fullerene $\mathrm{C}_{60}$ anchoring on amine groups has been observed and shown in a previous work ${ }^{6}$. This is particularly challenging for molecular electronics since electronic properties of such $\pi$-conjugated molecules enable their application in various molecular devices ${ }^{7}$. Among potential applications, such a bottom-up approach with a two step grafting is very attractive to build $\mathrm{C}_{60}$-based capacitive memory cells with improved control of the density of active storage sites compared to the more developed top-down approach ${ }^{8}$. However, the experimental study of such complex nanostructures is extremely difficult. Indeed, during the formation of PATP SAMs some molecules may be linked to gold via amine groups although less favourable than linking via the thiol function ${ }^{9}$, and further grafting process of fullerenes may modify the organization of the pre-formed PATP SAM. Among all characterisation tools, Surface Enhanced Raman Spectroscopy (SERS) is extremely interesting as it can bring different information: metal-molecules interaction, charge transfer mechanism, electronic properties, molecule orientation, and so forth. This spectroscopy is based on the giant electromagnetism enhancement observed in the near field of metallic nanoparticle such as gold or silver ${ }^{10}$ through a plasmonic mechanism. It is generally admitted that an additional feature, usually called the chemical enhancement, also contributes to the overall SERS signal. This last contribution is subject of many debates in the SERS community and its exact role remains unclear. In the case of P-aminothiophenol (PATP) SAM on gold or silver nanostructures, SERS has been extensively used ${ }^{11-14}$. Due to the strong affinity of the thiol group of this molecule for metals, SERS measurements can be extremely easily performed. All previous studies have shown that a charge transfer (CT) between this molecule and the metal occurs: in the SERS spectrum this CT gives rise to strong evolution in the Raman modes, but the exact origin of this mechanism remains unclear ${ }^{15}$. It must also be said that this molecule has been studied in complex metal-PATP-metal sandwich nanostructures ${ }^{12}$ using SERS too. In the case of $\mathrm{C}_{60}$ molecules, SERS studies are much less numerous ${ }^{16}$ : this molecule has no functional group with a strong affinity for metals and it is thus almost impossible to prepare a clean bundle-free thin film of $\mathrm{C}_{60}$ like a SAM directly on the metal. A possible technique is to use an intermediate molecule to connect $\mathrm{C}_{60}$ to the metal ${ }^{17}$, as afore discussed with PATP. It must be noticed that most of $\mathrm{C}_{60}$ SERS studies 
have been performed using $1064 \mathrm{~nm}$ excitation, which is quite far from the plasmon absorption of gold or silver nanostructures. This might explain why the connecting molecule is usually not clearly observed in such analyses. Thus, both PATP and $\mathrm{C}_{60}$ molecules have already been studied by SERS. Nevertheless, to our knowledge, few SERS studies have been performed using more complex SAM of stacked molecules. In this study we focused on the SERS characterisation of PATP and stacked PATP-C ${ }_{60}$ SAM prepared on gold. We clearly observed that the SERS spectrum of PATP strongly depends on two experimental parameters: the density of the layer and laser power. We observed a progressive but irreversible evolution of the modes under high power laser illumination. A possible chemical change of the PATP molecule is discussed. For PATP-C 60 SAM the SERS signal of both PATP and $\mathrm{C}_{60}$ was measured. A clear evolution of the SERS spectrum of PATP is observed, suggesting a change in the organisation of the PATP layer during $\mathrm{C}_{60}$ grafting. The evolution of the Raman modes is compared with X-ray photoelectron spectroscopy (XPS) of the carbon and nitrogen core levels.

\section{EXPERIMENTAL SECTION}

Gold wire (99,99\%) was obtained from Neyco. P-aminothiophenol (PATP, 97\%), and fullerene $\mathrm{C}_{60}$ (sublimated, 99.9\%) were purchased from Sigma-Aldrich. Anhydrous toluene (synthesis grade) and absolute ethanol (99.99\%) were obtained from Fisher Chemicals. Both molecules and solvents were used without further purification.

The nanostructured surfaces are prepared by thermally evaporating $15 \mathrm{~nm}$ of gold onto typical glass microscope slides in a vacuum chamber. Deposition is made at a rate of $\sim 1 \AA . \mathrm{s}^{-1}$ under a vacuum pressure less than $5.10^{-6}$ mbar. Those nanostructured susbtrates are made of gold island films ${ }^{18}$ and have a low electrical conductivity. As a consequence for XPS measurements we used different gold substrates prepared with $\sim 200 \mathrm{~nm}$ of gold Joule evaporated $\left(\sim 1 \AA . \mathrm{s}^{-1}\right)$ on a thin chromium layer deposited on a silicon substrate. Gold substrates are then soaked for 12 hours in $1 \mathrm{mM}$ alcoholic solution of PATP. Substrates are then rinsed with pure ethanol to remove physisorbed molecules. $\mathrm{C}_{60}$ are grafted on the top of PATP monolayer under reflux conditions in toluene, at a temperature of $110^{\circ} \mathrm{C}$ and under nitrogen flow. Afterward, PATP+C 60 SAM are sonicated in pure toluene to remove physisorbed molecules. PATP molecules should have an almost standing up ${ }^{9}$ orientation with the thiol functional group linked to the gold surface, and $\mathrm{C}_{60}$ are adsorbed via the amino group, forming a two layer self-assembled structure. Few SERS studies have been performed on such nanostructures. It must be noticed that for a part of our samples we have observed locally an aggregation of $\mathrm{C}_{60}$ molecules (confirmed by Raman measurements) whose origin remains unclear. As a consequence we performed measurements out of those aggregation areas.

The XPS spectra were recorded in normal emission geometry with a $125 \mathrm{~mm}$ radius hemispherical analyzer (with a pass energy of $20 \mathrm{eV}$ ) and a $\mathrm{Al} \mathrm{K \alpha} \mathrm{X}$-ray source $(h v=1486.6 \mathrm{eV})$ in ultrahigh vacuum (UHV). The 
XPS spectra are referenced to the spectroscopic Fermi level, and no corrections have been made for any surface charging. The area intensities were obtained following the spectral fitting and integration with Voigtlike functions.

SERS measurements were performed using an Horiba Jobin Yvon HR800 spectrometer using $632.8 \mathrm{~nm}$ excitation. The laser was focused on the sample via a $50 \times$ objective. Typical laser power on the sample was $1.5 \mathrm{~mW}$ (D1 filter) or $150 \mu \mathrm{W}$ (D2 filter). Those powers insure a rather high signal to noise ratio with a reasonable acquisition time.

\section{RESULTS AND DISCUSSION}

The SERS signal of PATP deposited on metallic nanostructures has already been extensively studied. It has now been clearly established that this spectrum is different from the bulk one, in opposition with what is commonly observed for dye molecules (methylene blue, rhodamine etc..). This feature has been interpreted as a sign of a charge transfer mechanism via an Herzberg Teller vibronic coupling ${ }^{13,19,20}$. However the full interpretation of the observed modes has led to many discussions in the literature and the exact origin of the enhancement is still under debate. In table one is reported the vibrational assignment of the observed Raman of PATP according to different studies. It can be clearly seen that this attribution remains unclear. In addition, a recent theoretical study has concluded that the abnormal

\begin{tabular}{|c|c|c|c|c|c|c|}
\hline $\begin{array}{l}\text { Raman } \\
\text { modes of } \\
\text { ATPa }^{a}\end{array}$ & $\begin{array}{l}\text { SERS modes } \\
\text { of ATP } \\
\text { (high } \\
\text { concentration) }\end{array}$ & $\begin{array}{l}\text { SERS modes } \\
\text { of ATP } \\
\text { (low } \\
\text { concentration) }\end{array}$ & $\begin{array}{l}\text { SERS modes } \\
\text { of ATP after } \\
\text { annealing in } \\
\text { toluene }\end{array}$ & $\begin{array}{c}\text { SERS modes } \\
\text { of ATP and } \\
\mathrm{C}_{60}\end{array}$ & $\begin{array}{c}\text { Vibrational } \\
\text { assignmenta, }\end{array}$ & $\begin{array}{c}\text { Vibrational } \\
\text { assignment }\end{array}$ \\
\hline $1093 \mathrm{~cm}^{-1}$ & $1075 \mathrm{~cm}^{-1}$ & $1075 \mathrm{~cm}^{-1}$ & $1075 \mathrm{~cm}^{-1}$ & $1075 \mathrm{~cm}^{-1}$ & $\begin{array}{l}\mathrm{a}_{1} \text { mode from } \\
\text { ATP } \\
\mathrm{SC} \text { stretching } \\
+\mathrm{NH}_{2} \text { rocking }\end{array}$ & $\begin{array}{c}\text { ATP } \\
\text { SC stretching }\end{array}$ \\
\hline $1171 \mathrm{~cm}^{-1}$ & $1140 \mathrm{~cm}^{-1}$ & $\begin{array}{c}1140 \mathrm{~cm}^{-1} \\
\text { (very weak or } \\
\text { not observed) }\end{array}$ & Not observed & Not observed & $\begin{array}{c}\mathrm{b}_{2} \text { mode from } \\
\text { ATP } \\
\mathrm{CH} \text { bending }\end{array}$ & $\begin{array}{c}\text { ATP } \\
\mathrm{CH} \text { bending }\end{array}$ \\
\hline $1208 \mathrm{~cm}^{-1}$ & $1180 \mathrm{~cm}^{-1}$ & $\begin{array}{c}1180 \mathrm{~cm}^{-1} \\
\text { (very weak or } \\
\text { not observed) }\end{array}$ & Not observed & Not observed & $\begin{array}{l}\mathrm{CN} \text { stretching } \\
+\mathrm{CH} \text { rocking }\end{array}$ & $\begin{array}{c}\text { ATP } \\
\mathrm{CH} \text { bending }\end{array}$ \\
\hline Not observed & $1390 \mathrm{~cm}^{-1}$ & $\begin{array}{c}1390 \mathrm{~cm}^{-1} \\
\text { (very weak or } \\
\text { not observed) }\end{array}$ & Not observed & Not observed & $\begin{array}{l}\mathrm{b}_{2} \text { mode from } \\
\text { ATP } \\
\mathrm{CC} \text { stretching } \\
+\mathrm{NH}_{2} \text { rocking } \\
+\mathrm{CH} \text { rocking }\end{array}$ & $\begin{array}{c}\text { ATP } \\
\text { Phenyl ring } \\
\text { deformation + } \\
\mathrm{NH}_{2} \text { rocking }\end{array}$ \\
\hline Not observed & $1435 \mathrm{~cm}^{-1}$ & $\begin{array}{c}1435 \mathrm{~cm}^{-1} \\
\text { (very weak or } \\
\text { not observed) }\end{array}$ & $1435 \mathrm{~cm}^{-1}$ & $1435 \mathrm{~cm}^{-1}$ & $\begin{array}{l}\mathrm{b}_{2} \text { mode from } \\
\text { ATP } \\
\mathrm{CC} \text { stretching } \\
+\mathrm{NH}_{2} \text { rocking }\end{array}$ & $\begin{array}{c}\text { ATP } \\
\text { Phenyl ring } \\
\text { deformation + } \\
\text { CN stretching }\end{array}$ \\
\hline Not observed & Not observed & Not observed & Not observed & $1460 \mathrm{~cm}^{-1}$ & $\begin{array}{c}\text { Mode from } \\
\mathrm{C}_{60}\end{array}$ & $\begin{array}{c}\text { Mode from } \\
\mathrm{C}_{60}\end{array}$ \\
\hline $1596 \mathrm{~cm}^{-1}$ & $1575 \mathrm{~cm}^{-1}$ & $1575 \mathrm{~cm}^{-1}$ & $1575 \mathrm{~cm}^{-1}$ & $1575 \mathrm{~cm}^{-1}$ & $\begin{array}{l}\mathrm{a}_{1} \text { or } \mathrm{b}_{2} \text { mode } \\
\text { from ATP } \\
\mathrm{CC} \text { stretching } \\
+\mathrm{NH}_{2} \\
\text { bending }\end{array}$ & $\begin{array}{c}\text { ATP } \\
\text { CC stretching } \\
\text { in phenyl ring } \\
+\mathrm{NH}_{2} \\
\text { bending }\end{array}$ \\
\hline
\end{tabular}

Table 1: list of most intense SERS and Raman modes of PATP SAM.

$a$ is $\operatorname{Ref}[13], b$ Ref [19] and c Ref [20]. 
enhanced peaks observed in SERS did not originate from PATP molecule itself ${ }^{15}$. As a consequence the complete SERS analysis of PATP SAMs is still necessary. In figure 1 are shown SERS spectra for two different densities of PATP molecules deposited on nanostructured gold. The high density layer is obtained after $12 \mathrm{~h}$ dipping in a $1 \mathrm{mM}$ PATP solution whereas the low density layer is obtained after a one minute dipping in a $10^{-5} \mathrm{~mol} / \mathrm{L}$ solution. For both layers, two spectra were measured: the first one with a $1.5 \mathrm{~mW}$ excitation, the second one with a $150 \mu \mathrm{W}$. According to classical Raman theory, the spectrum should not depend on the density of the PATP layer, except for its global intensity. Surprisingly it is not what is observed in figure 1 . Some Raman modes $\left(1140,1185,1390\right.$ and $1435 \mathrm{~cm}^{-1}$, mainly $b_{2}$ modes according to table 1) are clearly not observed or have a much lower intensity when the layer density is low. In addition, the intensity of some modes also depends on laser power, in particular for the high density layer.

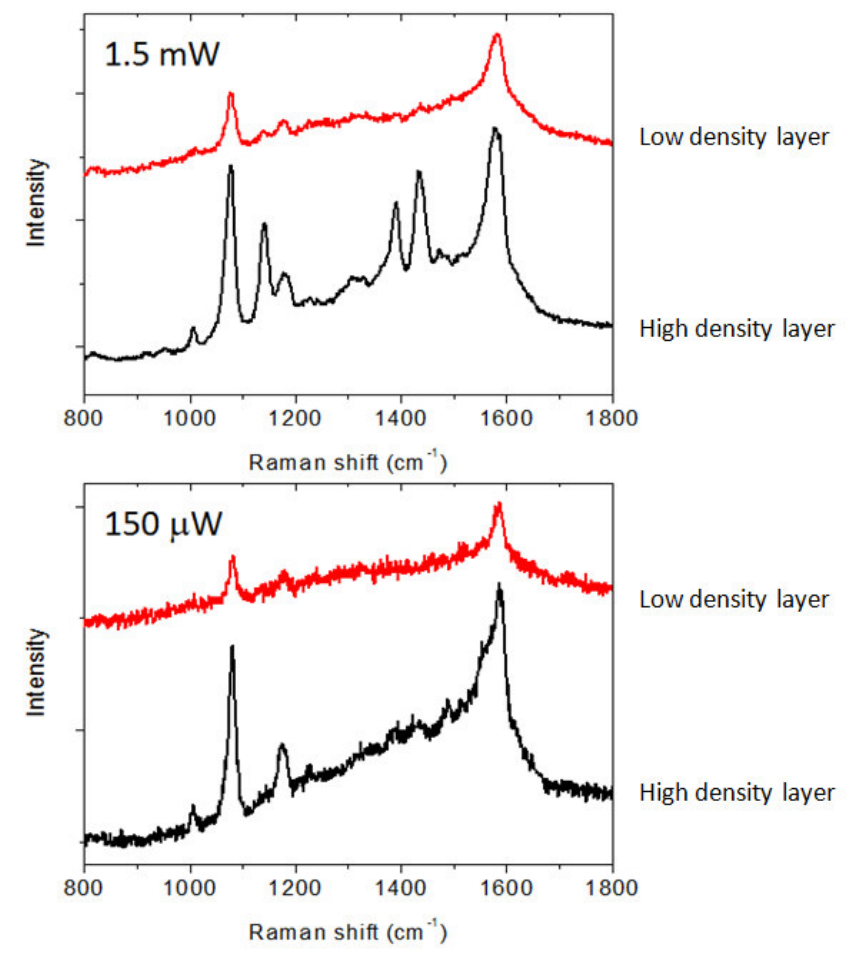

Figure 1: SERS spectra of low density layer (red) and high density layer (black) of PATP for two laser excitations: top $1.5 \mathrm{~mW}$, bottom $150 \mu \mathrm{W}$. Wavelength: $632.8 \mathrm{~nm}$

It can immediately be remarked that Raman modes with pure $b_{2}$ symmetry are most affected by the density of the layer. According to spectra shown in figure 1 these modes are also most affected by laser power. As a first conclusion it appears clearly that the observed modes and their relative intensity in the SERS spectrum of PATP strongly depend on the experimental conditions. According to our measurements at least two factors can modify this spectrum: laser power and layer density. To gain better understanding on the influence of laser power we acquired short time spectra (1s) of the high density layer under a $1.5 \mathrm{~mW}$ laser illumination. Two spectra are shown in figure 2: one immediately after turning on the laser, and the other 
after 30s of illumination. It appears clearly that an evolution of the SERS spectrum occurs during laser illumination. At the beginning the spectrum is similar to what is observed with low density layer or low power illumination. But after 30s the spectrum is different, and the intensity of $b_{2}$ modes $(1140,1185,1390$ and $1435 \mathrm{~cm}^{-1}$ ) has considerably increased, giving rise to a spectrum similar to that in figure 1 . As a consequence, it seems that SERS spectrum of PATP strongly depends on experimental conditions. It appears clearly that with low power laser illumination, $b_{2}$ modes are not enhanced, in accordance with recently published theoretical predictions ${ }^{15}$. The question is why the PATP SERS spectrum is progressively modified when laser power is increased. This evolution of PATP modes with laser illumination has already been reported by Kim et al. ${ }^{14}$ and interpreted as a consequence of threshold energy in the charge transfer enhancement. This feature should occur whatever the density of the PATP layer: as can be seen in figure 1, the low density layer has low $b_{2}$ modes even if laser power is high. As this threshold energy should reasonably not depend on the layer density this hypothesis cannot be considered for the observed evolution of Raman modes. Another possible explanation is a progressive degradation or modification of the PATP molecule induced by laser heating. Degradation is usually observed in Raman measurements when laser power density is too high. In this case, new Raman modes are present and the characteristic modes of the molecules disappear. As can be seen in figure 2 this is not what we observed: after a few seconds of laser illumination, the original modes of PATP are still present and their intensity has not decreased. This suggests that PATP is not totally damaged but it does not exclude a partial modification of the molecule as we clearly observed an evolution of the relative intensity between the different Raman modes of PATP. In previous studies the evolution of the $b_{2}$ modes was interpreted as a sign of the charge transfer, once again this mechanism should reasonably not depend on laser power and layer density. We can also assume that a change of the molecule orientation is induced by laser heating. This orientation change should modify the selection rules, lying to the observation of new modes. SERS has already been used to determine the orientation of $\mathrm{C}_{2 v}$ molecules adsorbed on metals: phthalazine ${ }^{21}$, benzoic acid $^{22}$, thiophenol ${ }^{23} \ldots$. In the case of PATP, it has been demonstrated that this molecule is oriented with the $\mathrm{C}_{2}$ axis almost normal to the surface. With this standing up orientation, electromagnetic enhancement theory cannot explain all the observed modes in the SERS spectrum of this molecule and chemical enhancement is introduced ${ }^{19}$. To explain the observed evolution of SERS modes of PATP we should suppose that under strong laser illumination this orientation changes, giving rise to new modes with different selection rules. This hypothesis appears as highly improbable. As a first conclusion, the most reasonable hypothesis for the evolution of the PATP modes with laser power and layer density is a chemical modification of the PATP molecules, excluding a total degradation of the molecule, as only modes reported in table 1 are observed. The dimerization of PATP molecules has already been reported on gold electrodes via an electrochemical mechanism ${ }^{24}$. In addition, some recent studies ${ }^{25}$ have suggested the production of dimercaptoazobenzene (DMAB) from PATP by catalytic coupling reaction on silver nanoparticles. This hypothesis has been advanced by Huang et al. to explain the evolution of PATP SERS modes ${ }^{26}$, the supposed $b_{2}$ modes being in fact modes of DMAB. As can be seen in figures 1 and 2, we observed similar features. As a consequence, we suggest that the reported 
evolution of SERS modes during high power laser illumination is induced by the transformation of PATP into a different molecule, certainly DMAB. This dimerization is not observed with low density layers as this reaction cannot occur if PATP molecules are too far one from the others.

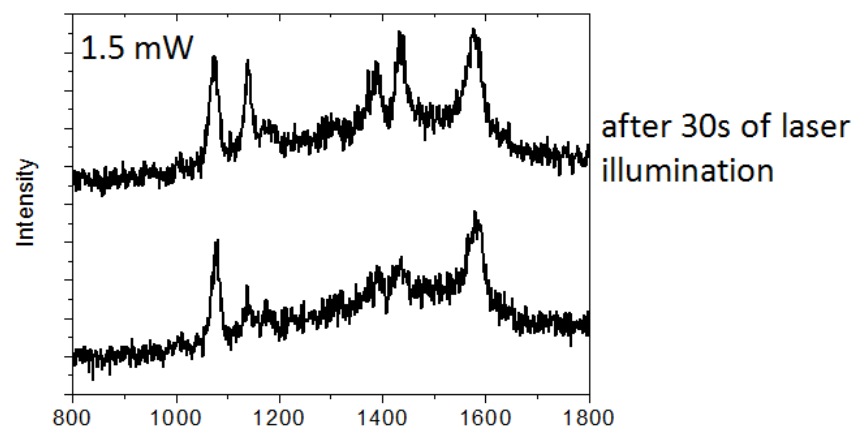

Figure 2: SERS spectra of a high density layer of PATP under a $1.5 \mathrm{~mW}$ laser illumination. The bottom spectrum is obtained immediately after the laser is turned on, the above spectrum is obtained 30s later. Wavelength: $632.8 \mathrm{~nm}$ Acquisition time: $1 \mathrm{~s}$

As explained in the introduction, PATP SAM can be interestingly used to graft fullerene molecules and obtain stacked SAM. The latter should then allow to obtain no more than one monolayer of $\mathrm{C}_{60}$, with a surface density that could be varied depending on the targeted applications (e.g. isolated $\mathrm{C}_{60}$ bundles for memory cells). In figure 3 are shown the C1s and N1s core level XPS spectra for a PATP layer before and after $\mathrm{C}_{60}$ grafting. The effective grafting of the PATP layer on the gold surface is demonstrated by the wellresolved N1s peak corresponding to the amino group (binding energy of $399.2 \mathrm{eV}$, full width at halfmaximum (FWHM) of $2.1 \mathrm{eV}$ ). Remarkably, no binding energy peak usually attributed to $\mathrm{N}$ atoms involved in hydrogen bonds ${ }^{27,28}$ is observed, thus suggesting a low order in the organic layer or a good cleanliness of the film. The $\mathrm{C} 1 \mathrm{~s}$ spectrum is mainly composed of an intense peak at $284.3 \mathrm{eV}$ binding energy corresponding to $\mathrm{C}-\mathrm{C}$ bonds and a minor peak around $286.3 \mathrm{eV}$ corresponding to carbon bonded to sulphur or nitrogen atoms. 

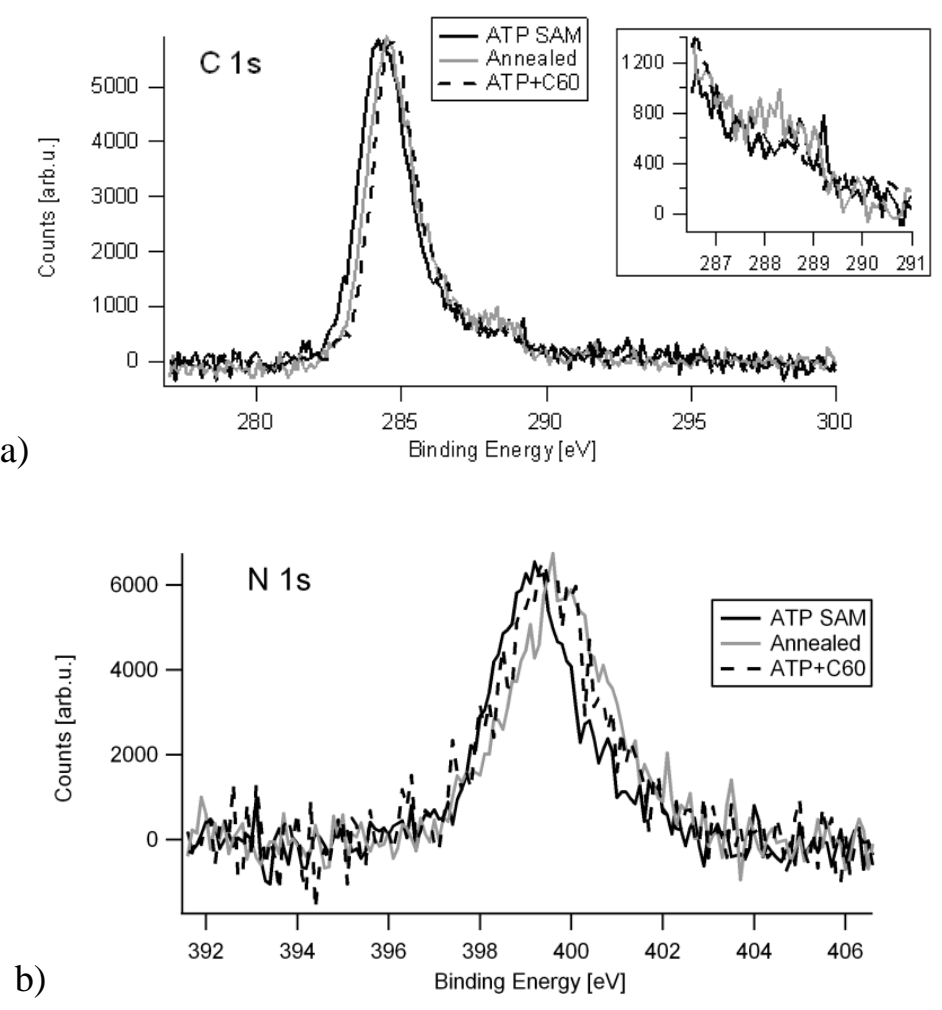

Figure 3: XPS spectra of the (a) C 1s and (b) N 1s core levels for different sample preparations (see text for details).

After $\mathrm{C}_{60}$ deposition XPS spectra are slightly modified. While the $\mathrm{C} 1 \mathrm{~s}$ spectrum remains nearly identical to that of PATP SAM, the nitrogen N1s peak is now centred at $399.5 \mathrm{eV}$ and slightly broader (FWHM $2.5 \mathrm{eV}$ ). Such feature can be induced by the reaction of $\mathrm{C}_{60}$ molecules with the amino functional group or a change in the organisation of the PATP layer. No peak related to the formation of $\mathrm{N}$ bonded to $\mathrm{C}_{60}$ (binding energy above $400 \mathrm{eV}^{27}$ ) is observed, indicating a relatively low efficiency of the $\mathrm{C}_{60}$ grafting. In addition the quantitative analysis of the N/Au ratios indicates that the number of PATP molecules remained constant. A typical SERS spectrum after $\mathrm{C}_{60}$ adsorption is shown in figure 4 (black spectrum). Those spectra were obtained using $1.5 \mathrm{~mW}$ excitation (for lower laser power, the intensity is too low for a quantitative analysis). Comparing to spectra shown in figure 1 , a global decrease of signal intensity is observed ${ }^{29}$. Since the amount of PATP does not change after $\mathrm{C}_{60}$ adsorption, this decrease cannot be induced by a lower amount of PATP molecules. We cannot exclude that a part of gold nanoparticles are desorbed during $\mathrm{C}_{60}$ grafting decreasing the electromagnetic enhancement and the SERS signal. In final only 3 Raman modes of PATP are clearly observed: 1075, 1435 (weak intensity) and $1575 \mathrm{~cm}^{-1}$. In addition a very broad band between 1400 and 1600 $\mathrm{cm}^{-1}$ is superposed to those Raman modes, probably associated to impurities. Moreover a new Raman mode, with a rather weak intensity, is observed at $1460 \mathrm{~cm}^{-1}$. This mode is characteristic of $\mathrm{C}_{60}$ (as can be seen in 
the green spectrum of figure 4) and clearly confirms that $\mathrm{C}_{60}$ molecules have been adsorbed on PATP molecules. The weak intensity of this mode is rather surprising since in previous studies from the literature the intensity of $\mathrm{C}_{60}$ modes excited at $1064 \mathrm{~nm}$ was extremely intense. But it must be noticed that this IR excitation is quite far from plasmonic absorption of gold or silver nanostructures. Further confirmation of the presence of C60 molecules was also brought by UV-Visible optical absorption (1-Transmission) measurements ${ }^{30}$.
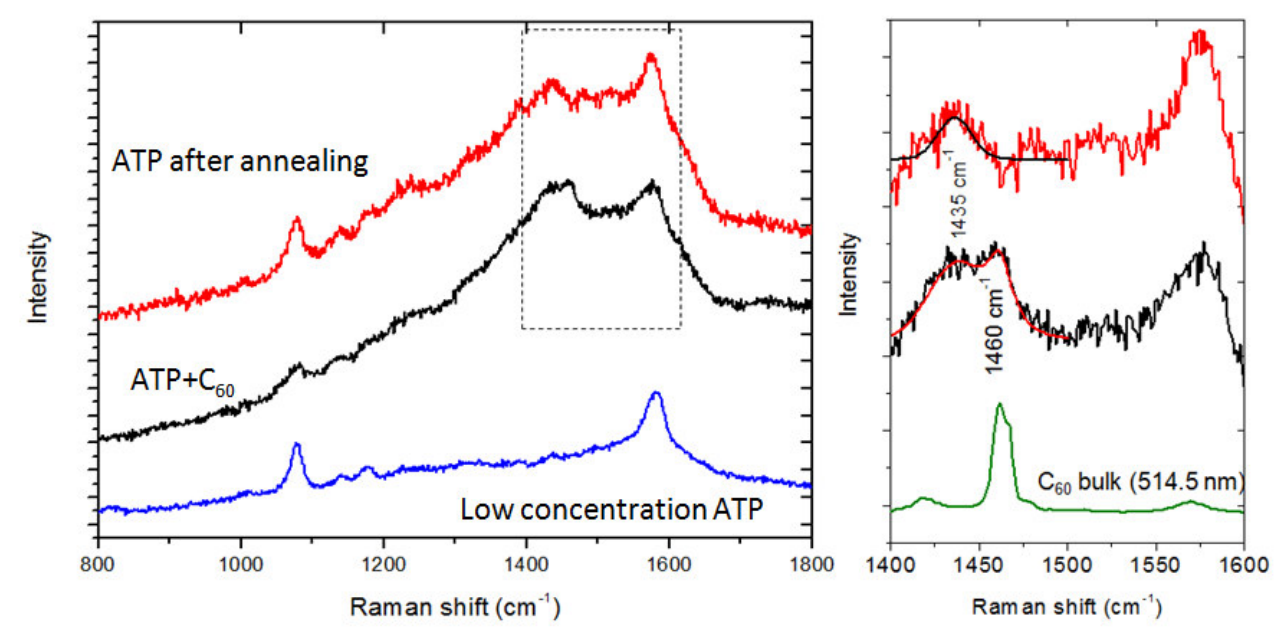

Figure 4: Left: SERS signal of PATP $+\mathrm{C}_{60}$ SAM (black), PATP after annealing in toluene (red) and low concentration PATP SAM ( $10^{-5} \mathrm{~mol} / \mathrm{L}, 1$ minute dipping in blue). Right: an enlargement of black and red spectra. The characteristic peak of $C_{60}$ at $1460 \mathrm{~cm}^{-1}$ can be clearly observed. The bulk spectrum of $\mathrm{C}_{60}$ obtained with $514.5 \mathrm{~nm}$ excitation is shown in green. For all other spectra, the wavelength excitation is $632.8 \mathrm{~nm}$ with a typical laser power of $1.5 \mathrm{~mW}$.

The question is why the PATP SERS spectrum is modified after $\mathrm{C}_{60}$ adsorption. Two hypotheses can be put forward: those modifications are induced by $\mathrm{C}_{60}$ adsorption itself or by the heating process operated to promote the adsorption. This high temperature treatment in toluene can induce a desorption and a reorganisation of the PATP layer due to the labile nature of the thiol to gold bonding. However, XPS measurements suggest that no PATP molecule desorbed during $\mathrm{C}_{60}$ adsorption. To elucidate the effect of $\mathrm{C}_{60}$ grafting, we put an Au-PATP sample in a toluene solution heated at the temperature used in the grafting process of $\mathrm{C}_{60}\left(\sim 110^{\circ} \mathrm{C}\right)$ but without dissolved $\mathrm{C}_{60}$ molecules. The XPS spectra of this sample can be seen in figure 3. The spectra of the nitrogen and carbon core levels are almost identical to the case of the $\mathrm{C}_{60}$ grafted sample. This important result suggests that the broadening of the nitrogen peak previously mentioned is certainly induced by a change in the organisation of the PATP layer due to the annealing process ${ }^{31}$. Concerning the $\mathrm{C} 1 \mathrm{~s}$ peak, one can note a small high binding energy shoulder at around $288 \mathrm{eV}$ that is slightly decreased after $\mathrm{C}_{60}$ deposition. This peak may be induced by the $\mathrm{C}=\mathrm{O}$ bond of an amide formed by reaction between amine and carbon dioxide ${ }^{32}$ and whose presence was detected in infrared spectra. The decrease of this signal after $\mathrm{C}_{60}$ deposition is consistent with the grafting of $\mathrm{C}_{60}$ that prevents from the 
formation of amide groups. The area of the carbon peak for the $\mathrm{C}_{60}$ grafted sample has increased by a factor of 1.7 as compared to the peak for the PATP SAM. This clearly indicates that $\mathrm{C}_{60}$ molecules have actually been fixed on the PATP SAM. More surprisingly the intensity of this same carbon peak is also higher for the sample after heating in toluene (by a factor of 1.2 as compared to the peak for the PATP SAM). Certainly carbon impurities are deposited on the surface during heating in toluene. Comparing the intensity of the carbon peak between the $\mathrm{C}_{60}$ grafted sample and the PATP sample heated in toluene, we can estimate the amount of $\mathrm{C}_{60}$ molecules grafted if we consider that the same amount of impurities was deposited. The effective increase of the $\mathrm{C} / \mathrm{N}$ ratio of $1.7-1.2=0.5 \mathrm{C} / \mathrm{N}$ corresponds to about 3 additional carbons for each PATP molecule, or one $\mathrm{C}_{60}$ molecule deposited every 20 PATP molecules on the surface. This corresponds roughly to about half of the maximal $C_{60}$ density achievable on a $(\sqrt{3} x \sqrt{ } 3) R 30^{\circ}$ PATP SAM, even if this organisation has not been clearly confirmed for PATP molecules on gold $^{33}$. As a consequence the density of $\mathrm{C}_{60}$ molecule is rather low, explaining why the $\mathrm{C}_{60}$ Raman peak has a rather weak intensity. This result also explains why the peak corresponding to nitrogen bonded to $\mathrm{C}_{60}$ was not observed in the XPS spectrum of figure 3: its absolute intensity is very low and the signal is furthermore screened by the presence of fullerene molecules. The SERS spectrum of a PATP SAM after heating in toluene is shown in figure 4 (red spectrum). Except for the $\mathrm{C}_{60}$ peak at $1460 \mathrm{~cm}^{-1}$, this spectrum is very similar to what we observed after $\mathrm{C}_{60}$ adsorption. This indicates that the evolution of Raman modes of PATP is induced by the high temperature treatment in toluene. We also performed a similar high temperature treatment without any solvent in an incubator and the spectrum was almost identical to the standard SERS spectrum of PATP, despite a slight decrease of intensity ${ }^{34}$. This indicates that it is not only temperature that plays a role in the evolution of PATP Raman modes, but also the presence of solvent. It must be noticed that both SERS spectra of $\mathrm{C}_{60}$ grafted sample and of PATP SAM annealed in toluene are very similar to the SERS spectrum of the low density PATP SAM in figure 1 . The only difference is the $1435 \mathrm{~cm}^{-1}$ mode, whose intensity is very weak for the low density PATP SAM. XPS measurements indicates that carbon impurities are adsorbed during the heating in toluene (intensity increase of the carbon mode), which is confirmed by the presence of a broad band between 1400 and $1600 \mathrm{~cm}^{-1}$ in the SERS spectrum ${ }^{35}$.

Different hypotheses may explain the evolution of PATP modes in SERS spectra. The first one is a possible rearrangement of gold nanoparticles induced by high temperature treatment. It is well known that annealing of metallic nanoparticles can bring modifications in their organisation (size, distribution, etc.) and can have very important consequences for SERS measurements ${ }^{36}$. With thiol molecules it is possible to create gold nanoparticle superlattices ${ }^{37}$. In the special case of PATP, this molecule can induce the formation of "sandwich" structures, that is to say interlinked nanoparticles via a PATP molecule. This has been reported in several studies ${ }^{12}$ and can have dramatic effects in the SERS spectrum of this molecule. Nevertheless, the formation of sandwich structures should prevent the adsorption of $\mathrm{C}_{60}$ in so far as both functional groups of PATP molecules should be already linked to gold. And as explicitly mentioned above, XPS and SERS measurements confirm the presence of $\mathrm{C}_{60}$ molecules. As a first conclusion we cannot totally exclude the possible formation of sandwich structures made of metallic nanoparticles interlinked by PATP molecules but 
the presence of $\mathrm{C}_{60}$ molecules rather suggests that most of PATP and $\mathrm{C}_{60}$ molecules are bound otherwise $\mathrm{C}_{60}$ should have been washed off the surface during the rinsing step. To explain the observed modifications of SERS spectra of PATP after $\mathrm{C}_{60}$ adsorption or high temperature treatment, another hypothesis can be proposed: a strong reorganisation of the PATP SAM. It is known that the stability under high temperature treatment of thiol SAM on gold (nanostructured or not) is limited ${ }^{38}$. Disorder, reconstruction and desorption can occur even at relatively moderate temperatures, typically around $100^{\circ} \mathrm{C}$. In addition, this stability strongly depends on the chemical structure of the adsorbed molecules ${ }^{39}$. The careful analysis of SERS spectra can bring information about the evolution of those SAM. Spectra reported in figure 4 are intermediate between the bulk and the SERS spectrum of PATP. They clearly show strong similarity with the low density modes, or the modes obtained with a low laser density. Once again only modes attributed to $b_{2}$ symmetry are affected. In previous SERS studies, the enhancement of those modes was attributed to a charge transfer mechanism based on a Herzberg-Teller contribution ${ }^{19,40}$, that is to say a chemical enhancement. More recently, Uetsuki et $a .^{41}$ have intercalated a thin spacer layer between the metal and the PATP molecules and have observed a strong decrease of the $b_{2}$ modes intensity at 1141,1390 and $1437 \mathrm{~cm}^{-1}$. With such sample, any possibility of chemical bonding between Au atoms and PATP molecules is completely eliminated and chemical enhancement is not expected, explaining the intensity decrease of those modes. In our experiments, we observed total disappearance of 1140 and $1390 \mathrm{~cm}^{-1}$ modes and a clear decrease of the $1437 \mathrm{~cm}^{-1}$ mode, whose intensity is now clearly lower than the $1575 \mathrm{~cm}^{-1}$ mode. This is very similar to what is reported by Uetsuki et al. This could suggest that chemical enhancement of PATP is also affected by the annealing in toluene and $\mathrm{C}_{60}$ adsorption. But as previously explained it is necessary to be very cautious with the chemical enhancement hypothesis due to the strong evolution of PATP modes with experimental parameters and the suggested dimerization of PATP molecules. In any case the strong similarity between the SERS spectrum of PATP annealed in toluene and the low density PATP layer suggests that this dimerisation do not occurs for those samples. In addition, the broadening and the shift of the N1s peak observed in XPS indicate that the layer is probably less organised. As a consequence we propose the following mechanism during $\mathrm{C}_{60}$ deposition: the annealing in toluene induces a desorption of PATP molecules, decreasing the layer density, and an introduction of carbon impurities. The layer organisation is also strongly decreased during this step. However no dimerization occurs and $\mathrm{C}_{60}$ molecules can be grafted on top of PATP molecules. It appears that this grafting is not very efficient as typically one $\mathrm{C}_{60}$ is grafted every 20 PATP molecules. This proposed mechanism is in accordance with all the reported experimental features: the evolution of the SERS modes (decrease of $b_{2}$ modes attributed to DMAB, appearance of broad modes between $1400 \mathrm{~cm}^{-1}$ and 1600 $\mathrm{cm}^{-1}$ ) and XPS spectra (broadening and shift of the peaks).

\section{CONCLUSIONS}

We studied the SERS signal from PATP and PATP-C 60 SAMs. We observed an evolution of the Raman signal of PATP depending on two parameters: the layer density and the laser power. It appears clearly that $b_{2}$ modes of PATP are not enhanced if one or both of these parameters is low, in accordance with theoretical 
predictions. In opposition, if these parameters are both high, the intensity of $b_{2}$ modes is enhanced. The evolution of these modes is associated to a dimerisation of PATP molecules into DMAB. For PATP-C $\mathrm{C}_{60}$ SAM we also observed strong evolution of the SERS spectra: $b_{2}$ modes have their intensity considerably decreased after $\mathrm{C}_{60}$ adsorption. Comparing SERS and XPS, we proposed a grafting reaction mechanism during $\mathrm{C}_{60}$ deposition in toluene. Impurities and disorganisation of the PATP layer are introduced, however those features do not prevent the adsorption of $\mathrm{C}_{60}$ molecules in a relatively low density, an issue that could be beneficial to build isolated discrete molecular storage sites for molecular memory cells. Our work demonstrates the capability of SERS for studying complex self-assembled layers on metallic nanostructures. Consequently, SERS could be envisaged as a challenging and powerful technique that could help better understanding electronic properties and processes involved within molecular devices.

\section{Acknowledgments}

This work was partly supported by the Conseil Général du Var and the Région ProvenceAlpes-Côte d'Azur. Funding from the Objectif 2 EEC programme involving the FEDER, the "Conseil Général du Var" Council, the PACA Regional Council, Toulon Provence Méditerranée, and ISEN-Toulon is acknowledged. The authors would like to thank Professor Valmalette for fruitful discussion and D. Goguenheim for his support. This work was also partly funded by the Agence Nationale de la Recherche (ANR, CARIOCA project number 2010-JCJC-918-01).

\section{References}

1 T. Hasobe, H. Imahori, P. V. Kamat et al., Journal of the American Chemical Society 127 (4), 1216 (2005).

2 B. de Boer, M. M. Frank, Y. J. Chabal et al., Langmuir 20 (5), 1539 (2004).

3 A. R. Morrill, D. T. Duong, S. J. Lee et al., Chemical Physics Letters 473 (1-3), 116 (2009).

4 C. D. Keating, M. D. Musick, M. H. Keefe et al., Journal of Chemical Education 76 (7), 949 (1999).

$5 \quad$ G. P. Miller, Comptes Rendus Chimie 9 (7-8), 952 (2006); K. M. Chen, W. B. Caldwell, and C. A. Mirkin, Journal of the American Chemical Society 115 (3), 1193 (1993).

G. Delafosse, L. Patrone, and D. Goguenheim, J. Nanosci. Nanotechnol. 11 (doi:10.1166/jnn.2011.4289) (2011).

7 D. Guerin, S. Lenfant, S. Godey et al., Journal of Materials Chemistry 20 (13), 2680 (2010).

$8 \quad$ A. Kanwal and M. Chhowalla, Applied Physics Letters 89 (20) (2006); H. S. Majumdar, J. K. Baral,

R. Osterbacka et al., Organic Electronics 6 (4), 188 (2005).

9 A. Ulman, An introduction to ultrathin organic films: from Langmuir-Blodgett to self-assembly. (Academic Press, Boston, 1991). 

plasmonic effects. (Elsevier, 2009).

J. W. Zheng, Y. G. Zhou, X. W. Li et al., Langmuir 19 (3), 632 (2003); K. Kim and H. S. Lee, Journal of Physical Chemistry B 109 (40), 18929 (2005); D. F. Zhang, L. Y. Niu, L. Jiang et al., Journal of Physical Chemistry C 112 (41), 16011 (2008); K. Kim, J. K. Yoon, H. B. Lee et al., Langmuir 27 (8), 4526 (2011); K. Kim, J. Y. Choi, H. B. Lee et al., Journal of Chemical Physics 135 (12), 9 (2011).

K. Kim and J. K. Yoon, Journal of Physical Chemistry B 109 (44), 20731 (2005); Y. L. Wang, H. J. Chen, S. J. Dong et al., Journal of Chemical Physics 124 (7) (2006).

M. Baia, F. Toderas, L. Baia et al., Chemical Physics Letters 422 (1-3), 127 (2006).

K. Kim, D. Shin, H. B. Lee et al., Chemical Communications 47 (7), 2020 (2011).

L. B. Zhao, R. Huang, Y. F. Huang et al., Journal of Chemical Physics 135 (13), 11 (2011).

P. Verma, K. Yamada, H. Watanabe et al., Physical Review B 73 (4), 045416 (2006).

Z. Q. Niu and Y. Fang, Vibrational Spectroscopy 43 (2), 415 (2007); Y. Fang, Q. J. Huang, P. J. Wang et al., Chemical Physics Letters 381 (3-4), 255 (2003).

See Supplementary Material Document No.

M. Osawa, N. Matsuda, K. Yoshii et al., Journal of Physical Chemistry 98 (48), 12702 (1994).

D. Maniu, V. Chis, M. Baia et al., Journal of Optoelectronics and Advanced Materials 9 (3), 733 (2007).

M. Moskovits and J. S. Suh, Journal of Physical Chemistry 88 (23), 5526 (1984).

M. Pagannone, B. Fornari, and G. Mattei, Spectrochimica Acta Part a-Molecular and Biomolecular Spectroscopy 43 (5), 621 (1987).

K. T. Carron and L. G. Hurley, Journal of Physical Chemistry 95 (24), 9979 (1991); T. H. Joo, M. S. Kim, and K. Kim, Journal of Raman Spectroscopy 18 (1), 57 (1987).

J. Lukkari, K. Kleemola, M. Meretoja et al., Langmuir 14 (7), 1705 (1998).

Y. R. Fang, Y. Z. Li, H. X. Xu et al., Langmuir 26 (11), 7737 (2010); D. Y. Wu, X. M. Liu, Y. F. Huang et al., Journal of Physical Chemistry C 113 (42), 18212 (2009).

Y. F. Huang, H. P. Zhu, G. K. Liu et al., Journal of the American Chemical Society 132 (27), 9244 (2010).

R. R. Sahoo and A. Patnaik, Journal of Colloid and Interface Science 268 (1), 43 (2003); R. R. Sahoo and A. Patnaik, Applied Surface Science 245 (1-4), 26 (2005).

J. E. Baio, T. Weidner, J. Brison et al., Journal of Electron Spectroscopy and Related Phenomena 172 (1-3), 2 (2009).

See Supplementary Material Document No.

See Supplementary Material Document No.

“ The broadening toward higher binding energies may be due to NH3+ and/or hydrogen bonding between $\mathrm{NH} 2$ terminal groups. In the latter case, the disorder may weaken p-p interactions betwen 
phenyl rings that can be more tilted thus promoting the formation of hydrogen bonding between terminal amines."

N. Graf, E. Yegen, T. Gross et al., Surface Science 603 (18), 2849 (2009).

V. Batz, M. A. Schneeweiss, D. Kramer et al., Journal of Electroanalytical Chemistry 491 (1-2), 55 (2000).

See Supplementary Material Document No.

A. Kudelski and B. Pettinger, Chemical Physics Letters 321 (5-6), 356 (2000).

M. Suzuki, Y. Niidome, and S. Yamada, Colloids and Surfaces a-Physicochemical and Engineering Aspects 284, 388 (2006).

J. E. Martin, J. P. Wilcoxon, J. Odinek et al., Journal of Physical Chemistry B 104 (40), 9475 (2000).

A. Chandekar, S. K. Sengupta, and J. E. Whitten, Applied Surface Science 256 (9), 2742 (2010); X.

D. Xiao, B. Wang, C. Zhang et al., Surface Science 472 (1-2), 41 (2001); E. Delamarche, B. Michel,

H. Kang et al., Langmuir 10 (11), 4103 (1994).

T. Ishida, H. Fukushima, W. Mizutani et al., Langmuir 18 (1), 83 (2002).

J. R. Lombardi, R. L. Birke, T. H. Lu et al., Journal of Chemical Physics 84 (8), 4174 (1986).

K. Uetsuki, P. Verma, T. Yano et al., Journal of Physical Chemistry C 114 (16), 7515 (2010). 